\title{
Repulsive interaction and contrast inversion in noncontact atomic force microscopy imaging of adsorbates
}

\author{
P. Rahe, R. Bechstein, J. Schütte, F. Ostendorf, and A. Kühnle* \\ Fachbereich Physik, Universität Osnabrück, Barbarastraße 7, 49076 Osnabrück, Germany
}

(Received 7 March 2008; published 7 May 2008)

\begin{abstract}
To understand contrast formation in atomic resolution noncontact atomic force microscopy (NC-AFM), we investigate whether or not repulsive tip-sample interaction contributes to contrast formation. We relate attractive and repulsive interactions to contrast features depending on both oscillating amplitude and measured detuning. Simulations based on a Morse potential illustrate the mechanism behind contrast inversion due to repulsive interactions above an adsorbate on the surface. Experimental NC-AFM images of adsorbates on mica and $\mathrm{TiO}_{2}$ surfaces confirm our simulations. Furthermore, we discuss the influence of the topography feedback loop on contrast formation above adsorbates, which illustrates that data interpretation can become rather delicate for constant-detuning images.
\end{abstract}

DOI: 10.1103/PhysRevB.77.195410

PACS number(s): 68.37.Ps, 68.43.Fg

\section{INTRODUCTION}

Atomic force microscopy (AFM) as invented by Binnig et $a l .{ }^{1}$ has become an indispensable tool for investigating a wide range of surfaces including dielectric substrates. Noncontact atomic force microscopy (NC-AFM) facilitates atomic resolution imaging first demonstrated for the $\mathrm{Si}(111)-(7 \times 7)$ surface $^{2}$ and is now obtained on a routine basis. The mechanisms governing atomic contrast formation in NC-AFM imaging especially for semiconducting and insulating surfaces have been investigated by both experiment $^{3,4}$ and theory. ${ }^{5,6}$ Also, manipulation of individual molecules $^{7}$ and atoms ${ }^{8}$ has been achieved by using NCAFM. Especially when performing manipulation, it is important to clarify to what extent repulsive interactions are involved. ${ }^{7}$ A closely related phenomenon observed when decreasing the tip-sample distance is the contrast inversion especially above protruding features, which is frequently ascribed to the onset of repulsive interaction. ${ }^{7,9,10}$ Besides protruding features, contrast inversion has also been observed on bare surfaces such as $\mathrm{Au}(111)^{11}$ and $\mathrm{Si}(111)-(7$ $\times 7) .{ }^{12-14} \mathrm{~A}$ comprehensive analysis of the mechanisms behind contrast inversion requires means for differentiating whether the tip enters the repulsive regime. Moreover, feedback loop effects need to be considered when imaging in the constant-detuning mode as the feedback loop response may heavily influence contrast formation. ${ }^{13,15}$

In this paper, we present an analysis clarifying whether or not the cantilever tip enters the repulsive regime based on the measured frequency shift, $\Delta f(z)$, and the actual oscillation amplitude. This analysis is used for elucidating physical mechanisms behind contrast inversion observed in NC-AFM imaging. Contrast inversion is always associated with a crossing point in two different $\Delta f(z)$ curves. The existence of two $\Delta f(z)$ curves can, however, be of different origins. Chemically different species usually result in different $\Delta f(z)$ curves that may have crossovers. Besides different chemistries, a crossover in two $\Delta f(z)$ curves can also be obtained by considering two identical $\Delta f(z)$ curves that are shifted with respect to the tip-surface distance $z$. This situation is present when the tip is closer to certain surface sites compared to others as for adsorbates or step edges. In this case, contrast inversion is due to the fact that the tip is penetrating into the repulsive regime of the protruding surface site. We present constant-height images of protruding adsorbates on a mica surface, which illustrate this effect.

Moreover, as many NC-AFM images are taken in the constant-detuning mode, we present NC-AFM data of protruding adsorbates on a titania surface measured with slow feedback loop settings. Compared to the case of true constant-height images, these measurements are rather difficult to interpret as the feedback loop alters the original data. An unambiguous interpretation of NC-AFM images is, therefore, delicate when a feedback loop is involved.

\section{REPULSIVE INTERACTIONS}

In NC-AFM operated in the frequency modulation (FM) mode, a sharp tip mounted at the end of an oscillating cantilever senses forces acting between tip and surface atoms. The tip-surface interaction shifts the cantilever resonance frequency $f$ relative to the cantilever's eigenfrequency $f_{0}$. The frequency shift $\Delta f=f-f_{0}$ (also referred to as detuning) can be directly used as an imaging signal. A central question for the interpretation of FM NC-AFM images is whether or not the tip enters the repulsive regime of the tip-sample interaction at the lower turning point of the oscillating cantilever. We discuss this by considering a model interaction potential based on a Morse potential as given by

$$
V_{\text {Morse }}(z, \kappa, \sigma)=E_{\text {bond }}\left(2 e^{-\kappa(z-\sigma)}-e^{-2 \kappa(z-\sigma)}\right) .
$$

In this potential, $E_{\mathrm{bond}}, \sigma$, and $\kappa$ are the bonding energy, equilibrium distance, and decay length, respectively. The parameters for the Morse potential are chosen as proposed for silicon by Pérez et al. ${ }^{16}$ The resulting potential curve is shown in the red solid graph in Fig. 1(a).

Using this interaction potential, an analytical expression for the frequency shift can be obtained. In order to keep this discussion general, we calculate the normalized frequency shift $\gamma(z)$ rather than the detuning $\Delta f(z)$, which is obtained from $\Delta f(z)$ according to 


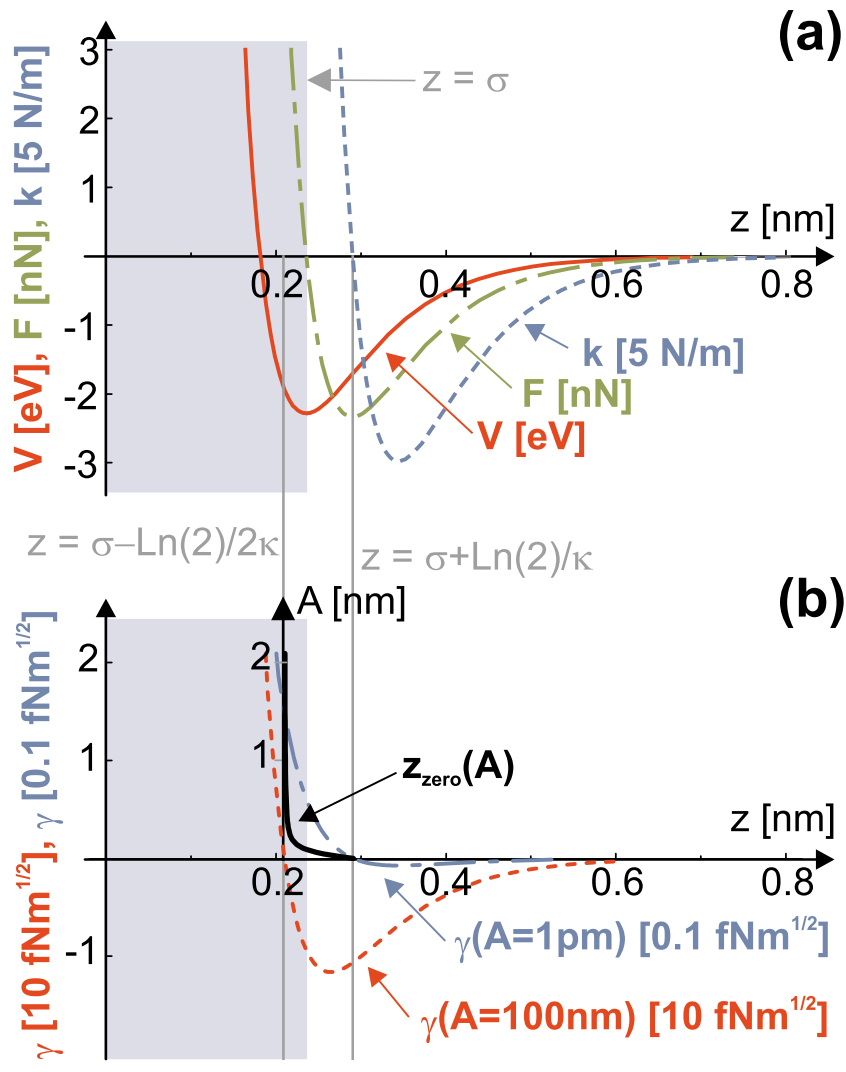

FIG. 1. (Color online) Relation between potential and normalized frequency shift curve in dependence on amplitude. (a) Potential (red, solid curve), force (green, dashed-dotted curve), and force gradient (blue, dashed curve) calculated by using a simple Morse potential and parameters for Si (Ref. 16). The shaded region is the repulsive regime. (b) The position of the zero of the normalized frequency shift curve is depicted in dependence on the amplitude $A$ (black, solid curve); the amplitude axis is drawn perpendicular to the $z$ axis. Corresponding normalized frequency shift curve for both large (red, dashed curve) and small (blue, dashed-dotted curve) amplitudes. Note that the scaling is different for the two amplitudes $\left(0.1\right.$ and $\left.10 \mathrm{fN} \mathrm{m}^{0.5}\right)$.

$$
\gamma(z, A)=\frac{k_{0} A^{3 / 2}}{f_{0}} \Delta f(z)
$$

where $A$ is the amplitude and $k_{0}$ is the stiffness of the cantilever. $^{17}$

The normalized frequency shift $\gamma_{x, y}(z, A)$ at a lateral position $(x, y)$ is given by ${ }^{18}$

$$
\gamma_{x, y}(z, A)=\frac{A^{3 / 2}}{\pi} \int_{-1}^{1} k_{x, y}[z-(q-1) A] \sqrt{1-q^{2}} d q
$$

where $k_{x, y}(z)$ is the force gradient and $z$ is the tip-surface distance at the lower turning point of the cantilever oscillation. Obtaining the force gradient by calculating the second derivative of the Morse potential, an analytical expression for the normalized frequency shift is given by ${ }^{19}$

$$
\begin{aligned}
\gamma(z, A)= & 2 \kappa E_{\mathrm{bond}} \sqrt{A}\left\{\operatorname { e x p } [ - \kappa ( z - \sigma ) ] \left[-M_{1}^{0.5}(-2 \kappa A)\right.\right. \\
& \left.+M_{2}^{1.5}(-2 \kappa A)\right]+\exp [-2 \kappa(z-\sigma)]\left[M_{1}^{0.5}(-4 \kappa A)\right. \\
& \left.\left.-M_{2}^{1.5}(-4 \kappa A)\right]\right\}
\end{aligned}
$$

where $M_{a}^{b}(x)$ is the Kummer function. ${ }^{20}$ Often, negative detuning values are associated with attractive interactions, while positive detunings are ascribed to repulsive interactions. To test the validity and limitations of this statement, we determine the zero $z_{\text {zero }}$ of the normalized frequency shift and compare $z_{\text {zero }}$ with the zero of the force, $F(\sigma)=0$, as the equilibrium distance $\sigma$ represents the border between the repulsive and the attractive interactions. The zero of the normalized frequency shift [Eq. (4)] is given by

$$
z_{\text {zero }}(A, \kappa, \sigma)=\sigma-A-\frac{1}{\kappa} \ln \left[\frac{I_{1}(A \kappa)}{I_{1}(2 A \kappa)}\right] .
$$

Here, $I_{n}(x)$ is the modified Bessel function. As can be seen from Eq. (5), the zero of the normalized frequency shift does depend on the amplitude $A$. This dependence of the zero of the normalized frequency shift on the amplitude is depicted in the black solid line in Fig. 1(b). The amplitude is added as an extra coordinate perpendicular to the $z$ axis. For large amplitudes, the zero is at a distance of $z_{\text {zero }}(A \rightarrow \infty, \kappa, \sigma)$ $=\sigma-\frac{\ln 2}{2 \kappa}$, while for small amplitudes, the zero shifts to larger tip-sample distances and converges to coincide with the zero of the force gradient at $z_{\text {zero }}(A \rightarrow 0, \kappa, \sigma)=\sigma+\frac{\ln 2}{\kappa}$.

The normalized frequency shift curves in the limits for $A \rightarrow \infty$ and $A \rightarrow 0$ are given in the red dashed $(A=100 \mathrm{~nm})$ and blue dashed-dotted $(A=1 \mathrm{pm})$ curves in Fig. 1(b), respectively. For each decay length $\kappa$, an amplitude $A_{0}$ exists with $z_{\text {zero }}\left(A_{0}, \kappa, \sigma\right)=\sigma$, which means that the minimum of the potential curve coincides with the zero of the normalized frequency shift curve. Only this particular amplitude guarantees that positive detunings imply repulsion while negative detunings correspond to attractive interaction. When a cantilever oscillating at a smaller amplitude than $A_{0}$ approaches the surface, the normalized frequency shift curve becomes positive before the lower turning point enters the repulsive regime. This means that measuring positive frequency shifts does not necessarily imply repulsive interaction when oscillating at small amplitudes. For amplitudes larger than $A_{0}$, in contrast, the normalized frequency shift only becomes positive when the tip is already within the repulsive regime at the lower turning point of the cantilever oscillation.

The differentiation between large and small amplitudes is given by the tip-sample interaction decay length $\kappa$. For the model force considered here, the onset of the repulsive regime coincides with the change in sign in the frequency shift for an oscillation amplitude of $A_{0} \approx 1.1 / \kappa \approx 0.8 \AA .^{21}$

Thus, for the amplitudes of $10 \mathrm{~nm}$ used in the experiments discussed below, we can conclude that measuring positive detunings necessarily implies repulsive interactions. On the other hand, measuring negative detunings does not guarantee that the cantilever solely oscillates in the attractive regime. 

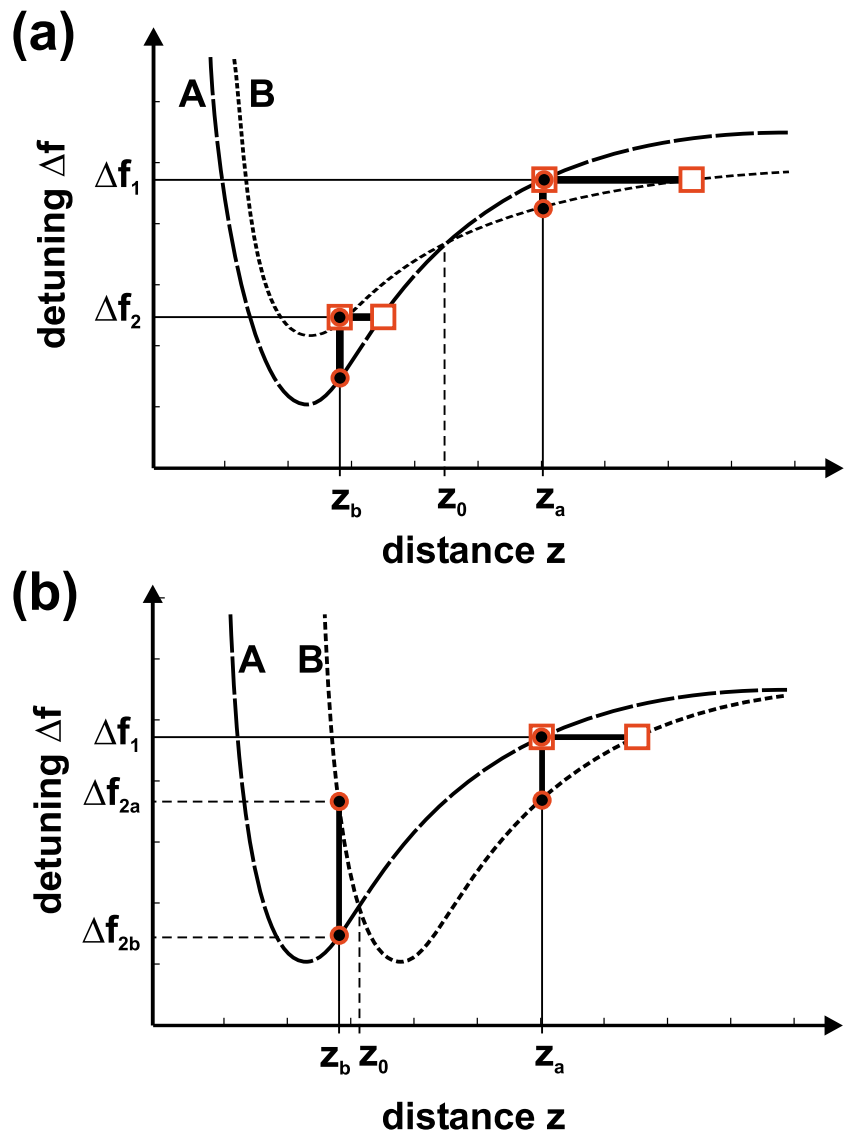

FIG. 2. (Color online) The origin of contrast inversion is a crossover in the $\Delta f(z)$ curves at two different sample sites $\mathbf{A}$ and $\mathbf{B}$. (a) Two $\Delta f(z)$ curves having a crossing point in the region of positive slope. Constant-height mode imaging at two tip-sample distances $z_{a}$ and $z_{b}$ is indicated by vertical lines. Constant-detuning imaging at two detuning values $\Delta f_{1}$ and $\Delta f_{2}$ is indicated by horizontal lines. The corresponding detuning values are marked by open circles and open squares, respectively. (b) Two identical $\Delta f(z)$ curves that are shifted in $z$, which result in a crossing point in the region of negative slope of the $\Delta f(z)$ curve of B. Again, constantheight mode imaging at two different tip-sample distances is indicated by vertical lines. Constant-detuning imaging is possible only at larger tip-sample distance as the region of inverted contrast cannot be reached due to feedback loop constraints.

\section{CONTRAST INVERSION}

Contrast inversion occurs when the $\Delta f(z)$ curves of the two different sample sites, $\mathbf{A}$ and $\mathbf{B}$, have a crossing point as such a crossing point implies that the order of the values of the $\Delta f(z)$ curves at different tip-sample distances is reversed. Two different $\Delta f(z)$ curves can originate from a different chemical nature of the two sample sites. Alternatively, chemically identical sample sites that have identical $\Delta f(z)$ curves can have a crossing point if the $\Delta f(z)$ curves are shifted in $z$, as would be the case for protruding sample sites or step edges.

In Fig. 2(a), a crossing point of two chemically different sample sites $\mathbf{A}$ and $\mathbf{B}$ is depicted in the region of positive slope in the $\Delta f(z)$ curves of both $\mathbf{A}$ and $\mathbf{B}$, i.e., the attractive regime of both sample sites as discussed above. If the mea- surement is carried out in constant-height mode, the tip is scanned with the distance feedback loop switched off and the detuning is detected at each point. This corresponds to the vertical lines in the graph in Fig. 2. At a tip-sample distance $z_{a}$, $\mathbf{B}$ will be imaged brighter than $\mathbf{A}$ because the negative frequency shift of $\mathbf{B}$ is larger than that of $\mathbf{A}$ due to a more attractive interaction (indicated by circles). At a distance $z_{b}$, however, the order is reversed: $\mathbf{A}$ will be imaged brighter than B. Furthermore, a distance $z_{0}$ exists, at which $\mathbf{A}$ and $\mathbf{B}$ are indistinguishable.

When measuring in constant-detuning mode, a feedback loop regulates the tip-sample distance for keeping the detuning constant. Consequently, horizontal lines need to be considered in this case and the contrast is provided by the $z$ movement instead of the detuning signal. Contrast inversion can be observed in this mode as well. Defining the detuning set point as $\Delta f_{1}, \mathbf{B}$ is imaged brighter than $\mathbf{A}$, as the tipsample distance is larger at $\mathbf{B}$ than at $\mathbf{A}$. In contrast, if the set point is $\Delta f_{2}, \mathbf{A}$ is imaged brighter than $\mathbf{B}$, as depicted by open squares in Fig. 2(a). Thus, for a crossing point in the attractive regime of both sample sites, both constant-height and constant-detuning measurements are possible at both sides of the crossing point and yield a qualitatively same contrast.

Another scenario can be considered, as depicted in Fig. 2(b). Here, chemically identical sample sites are considered, one of which is protruding $(\mathbf{B})$. Such a protruding feature is simulated by shifting two identical $\Delta f(z)$ curves with respect to the $z$ direction, necessarily leading to a crossing point in the region of negative slope of the $\Delta f(z)$ curve of B. At a distance $z_{a}$, $\mathbf{B}$ is imaged brighter than $\mathbf{A}$. This holds for both the constant-height mode at $z_{a}$ (depicted by circles) and for the constant-detuning mode at $\Delta f_{1}$ (depicted by squares). At a tip-sample distance $z_{b}$, constant-height measurements reveal contrast inversion as $\mathbf{A}$ is imaged brighter than $\mathbf{B}$. Constant-detuning imaging is, however, not possible due to the fact that constant-detuning imaging can only be performed as long as the sign of the slope of the $\Delta f(z)$ curve does not change, otherwise the regulation would act in the opposite direction. This prohibits - as a matter of principlethe observation of contrast inversion in constant-detuning mode in the case of identical but shifted $\Delta f(z)$ curves, as depicted in Fig. 2(b). In particular, contrast inversion due to repulsive interaction cannot be observed in the constantdetuning mode for the case of a protruding but chemically identical sample site. Nevertheless, if a slow feedback loop is involved, the minimum in the $\Delta f(z)$ curve can be continuously passed. In this "pseudoconstant-detuning mode," contrast inversion might be observed even when the crossing point is in the region of negative slope. We stress, however, that the case of slow feedback loop settings is severely complicating the interpretation as will be discussed in more detail in Sec. V.

\section{SIMULATION}

To illustrate the above mentioned situation of contrast inversion due to repulsive interaction, we perform a simple model simulation, which predicts the contrast of constant- 


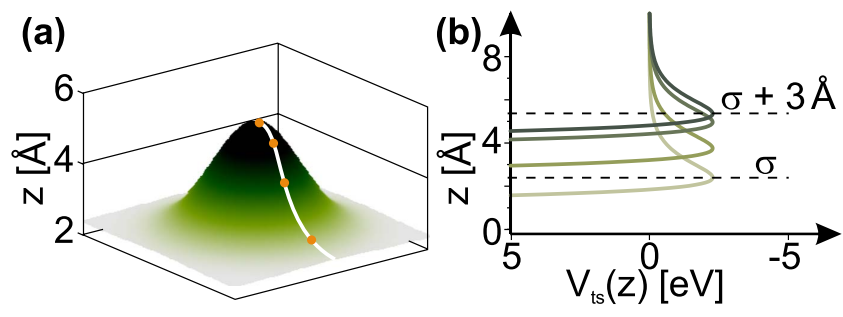

FIG. 3. (Color online) (a) Gaussian distribution of the equilibrium distance $\sigma_{x, y}$ and (b) several potential curves along the line at positions as indicated.

height images of a protruding adsorbate. Also, this simulation is based on a Morse potential as given in Eq. (1).

We simulate a generic protruding feature by modeling a Gaussian-shaped distribution of the Morse potential. This is done by substituting the equilibrium distance $\sigma$ in the Morse potential by a Gaussian distribution, $\sigma \rightarrow \sigma_{x, y}$,

$$
\sigma_{x, y}=\sigma+3 \AA \exp \left(-\frac{x^{2}+y^{2}}{D^{2}}\right),
$$

whereby $D$ reflects the lateral size of the feature. The minimum of the Morse potential at each point $(x, y)$ on the surface is shifted outward and the maximum shift of $3 \AA$ represents the center of the adsorbate, as shown in Fig. 3. We do not simulate individual atoms in the plane but use the above
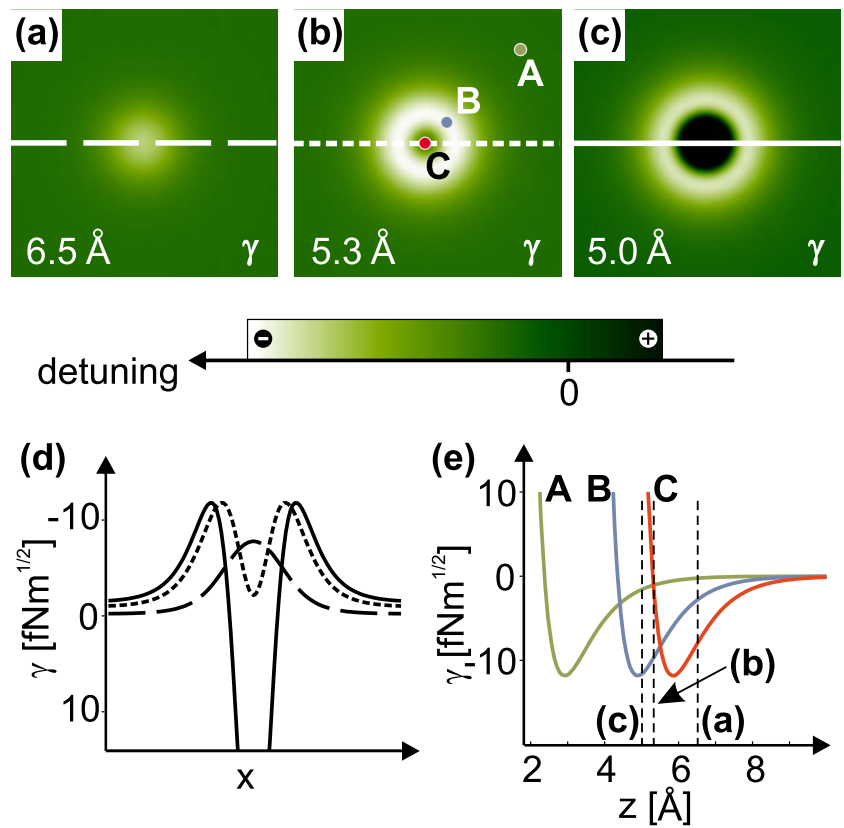

FIG. 4. (Color online) Results of the simulation, which show the evolution in imaging contrast upon gradually approaching the tip toward the sample from (a) to (c). Negative detuning is depicted bright while positive detuning is indicated dark. (a) Simulated constant-height image with a tip-sample distance of $6.5 \AA$ A. (b) Reduced tip-sample distance to $5.3 \AA$. (c) Constant-height image when further reducing the tip-sample distance to $5.0 \AA$. (d) Line profiles along the indicated lines in (a)-(c). (e) $\Delta f(z)$ curves at indicated positions $\mathbf{A}-\mathbf{C}$ in (b). defined potentials for describing the interaction of one lattice point with a single-atom tip.

As we numerically calculate, we discretize the $z$ axis by steps of $\Delta n=1 / N$ into $N$ parts and calculate the normalized frequency shift $\bar{\gamma}_{i, j}\left(z_{k}, A\right)$ analog to Eq. (3) for each position $(i, j)$ on the sample,

$$
\bar{\gamma}_{i, j}\left(z_{k}, A\right)=\frac{A^{3 / 2}}{\pi} \sum_{n \in \mathbf{I}} \bar{k}_{i, j}\left[z_{k}-(n-1) A\right] \sqrt{1-n^{2}} \Delta n,
$$

where $\mathbf{I}=\{m /(2 M) \mid m \in \mathbb{Z},-M \leq m \leq M\}$ and $M$ resembles the discretized amplitude.

By using a MATLAB code, we simulate constant-height images of a protruding adsorbate where the oscillation amplitude is $10 \mathrm{~nm}$. The results of the simulation are shown in Fig. 4. At larger distances between tip and sample ( $z$ $=6.5 \AA$ ), the adsorbate appears as a protrusion [Fig. 4(a)]. Upon approaching the tip toward the sample to $z=5.3 \AA$ [Fig. 4(b)], the negative frequency shift in the center is smaller compared to Fig. 4(a). Additionally, a bright rim evolves surrounding the center. Upon further reducing the tip-sample distance to $z=5.0 \AA$, the adsorbate center appears even darker while the rim remains as bright, as shown in Fig. 4(c). At this tip-sample distance, the frequency shift at the center of the adsorbate is positive, which illustrates that for such a large amplitude, the lower turning point of the cantilever is already in the repulsive regime above the protruding adsorbate [Fig. 4(d)]. This can be seen in Fig. 4(e), where the $\Delta f(z)$ curves at three different sample sites $\mathbf{A}-\mathbf{C}$ are shown. The corresponding heights at which the simulated images were obtained are indicated by dashed, vertical lines. From Fig. 4(e), the origin of the bright rim can be identified as the minimum of the $\Delta f(z)$ curves at a given tip-sample distance.

\section{EXPERIMENTS}

Simulations are tested against NC-AFM experiments on protruding adsorbates on mica and titania surfaces. Measurements on Muscovite mica are performed in a UHV chamber by using a UHV 350 NC-AFM from RHK (Troy, Michigan). The microscope is operated under UHV conditions with a base pressure below $10^{-10}$ mbar at room temperature. We use Si cantilevers from NanoWorld (Neuchâtel, Switzerland) with a resonance frequency of about $75 \mathrm{kHz}$ (type PPP$\mathrm{NCH}$ ) operated at an amplitude of about $10 \mathrm{~nm}$. The mica samples are of best available quality (Hi-Grade), which can be purchased from Plano GmbH (Wetzlar, Germany). They are cleaved in air, transferred into the chamber, and degased for $2 \mathrm{~h}$ at $580 \mathrm{~K}$. A bias voltage in the range of $\pm 2.5 \mathrm{~V}$ is applied to the sample for minimizing electrostatic interactions.

Measurements are performed in the constant-height mode by completely switching off the $z$ regulation. For the constant-height images presented here, we mention the "average detuning" instead of the detuning set point which is associated with constant-detuning images.

Measurements on a rutile $\mathrm{TiO}_{2}(110)$ surface are performed in a different UHV chamber equipped with a VT AFM 25 from Omicron (Taunusstein, Germany) and an easy- 

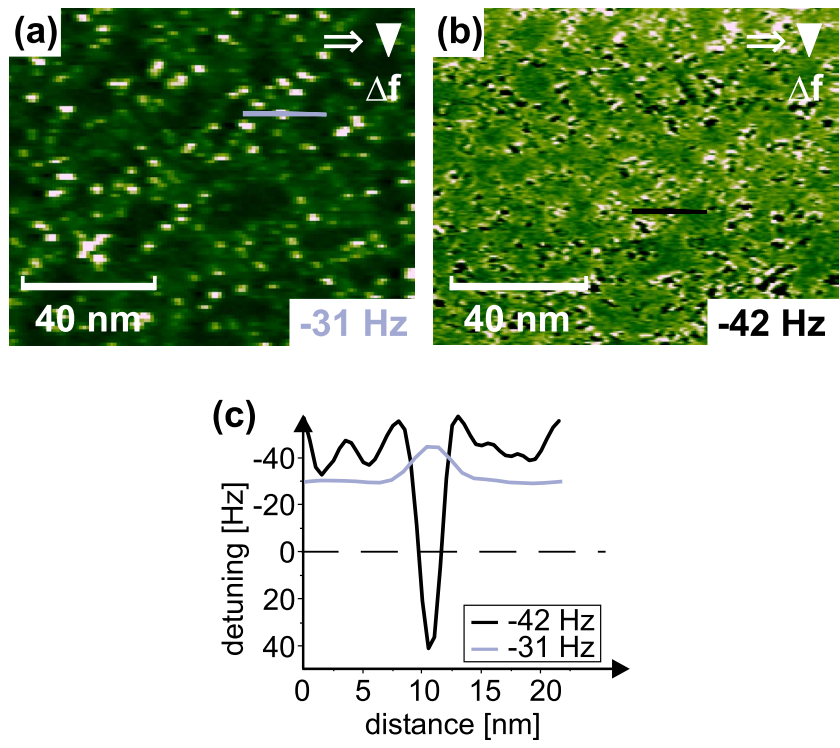

FIG. 5. (Color online) Detuning images of the mica surface. (a) Image obtained at an average detuning of $-31 \mathrm{~Hz}$, where protruding adsorbates are observed in this image, which are displayed as white spots due to a larger negative detuning. (b) Upon reducing the tip-sample distance (average detuning of $-42 \mathrm{~Hz}$ ), the adsorbates exhibit inner depressions. (c) The comparison of line plots taken over the adsorbate in (a) and (b) clarifies the contrast inversion. While protrusions are seen at an average detuning of $-31 \mathrm{~Hz}$, even positive detuning values are obtained in the center of the adsorbates for an average detuning of $-42 \mathrm{~Hz}$. The images are raw data, which are taken in constant-height mode.

PLL Plus from Nanosurf (Liestal, Switzerland) for oscillation excitation and signal demodulation. We use similar cantilevers (type PPP-NCH) with a resonance frequency of about $300 \mathrm{kHz}$ operated at an amplitude of about $10 \mathrm{~nm}$. For these experiments, the cantilevers are sputtered with $\mathrm{Ar}^{+}$ions after insertion into the chamber. The $\mathrm{TiO}_{2}$ samples are electron-polished crystals of the highest quality available from MTI (Richmond). The surface is cleaned by cycles of $\mathrm{Ar}^{+}$ion sputtering at $1 \mathrm{keV}$ and annealing at $900 \mathrm{~K}$. In order to minimize long-range electrostatic interactions, a bias voltage of $+1.54 \mathrm{~V}$ is applied to the tip.

In Fig. 5, images of the mica surface are shown, which are taken at the two different tip-sample distances. Upon cleavage in air, a dense layer of particles remains on the surface. These particles originate from a reaction of air-borne contaminants with surface potassium ions, as will be discussed in detail in a forthcoming publication. The shown images are raw detuning data; bright colors correspond to a large negative detuning (i.e., strong attractive interaction), whereas dark colors correspond to small negative detuning or even positive detuning (i.e., weak attractive or repulsive interaction).

In Fig. 5(a), a constant-height image is shown (average detuning of $-31 \mathrm{~Hz}$ ). The adsorbed species appear as protrusions. Upon approaching the tip toward the sample (average detuning of $-42 \mathrm{~Hz}$ ), the imaging contrast changes. Depressions with bright rims evolve, as shown in Fig. 5(b). It is important to note that this image contrast is reversible upon increasing the tip-sample distance again (not shown). These
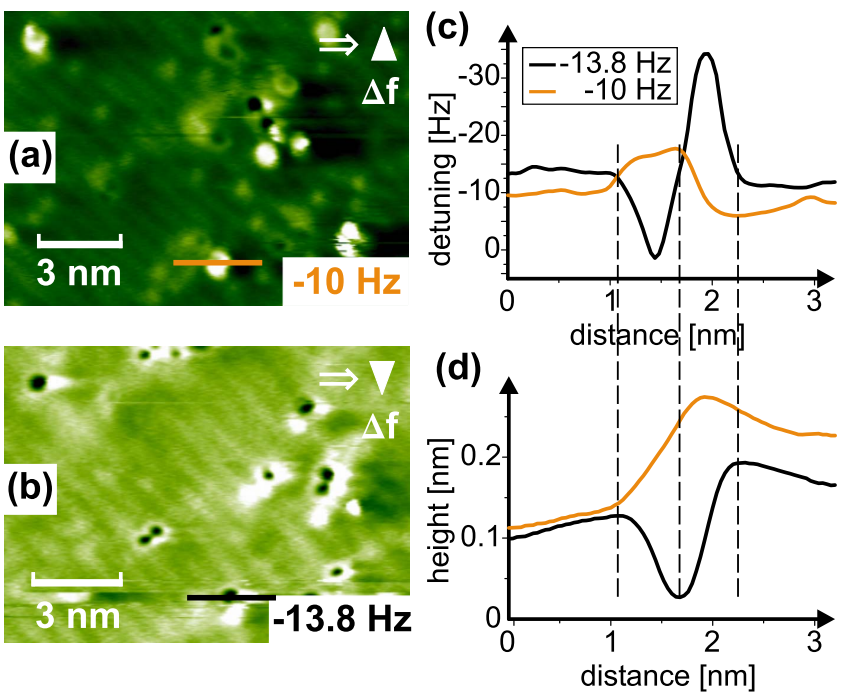

FIG. 6. (Color online) Images of the $\mathrm{TiO}_{2}(110)$ surface. (a) At an average detuning of $-10 \mathrm{~Hz}$, protruding features are observed. (b) Upon decreasing the tip-sample distance by increasing the average negative detuning to $-13.8 \mathrm{~Hz}$, some of the adsorbates are imaged with an inverted contrast, which reveal inner depressions surrounded by bright rims. (c) Line profiles taken from the detuning images (a) and (b) at the indicated positions. (d) At an average detuning set point of $-10 \mathrm{~Hz}$, the feedback regulation is able to follow a contour of nearly $\Delta f=$ const above the adsorbate. At a reduced tip-sample distance (average detuning of $-13.8 \mathrm{~Hz}$ ), the feedback further approaches the tip toward the sample because the measured detuning is smaller than the given set point. The presented images are raw data except for a plane correction of the $z$ data displayed in (d). The $z$ position has an additional, unknown offset. Several of the species seen in these images appear to diffuse on the surface. Moreover, chemically different species that exhibit contrast inversion at different tip-surface distances seem to exist.

images resemble the simulated images which are shown in Fig. 4. A comparison of line profiles above the adsorbates is given in Fig. 5(c), which confirms that the depression in the center of the species in Fig. 5(b) corresponds to positive detuning values. Based on the analysis discussed in Secs. II and IV, we can, therefore, conclude that the depression in the center of the adsorbates originates from repulsive tipadsorbate interactions.

A similar situation is demonstrated for adsorbates on titania, as shown in Fig. 6. Here, we also intend to study the influence of the topography feedback on apparent contrast features. Therefore, measurements are done in an intermediate mode by using the feedback loop with slow response.

On UHV-cleaned rutile $\mathrm{TiO}_{2}(110)$ oxygen vacancies, single hydroxyls as well as double hydroxyls are known defects in the bridging oxygen rows. ${ }^{22}$ The protruding features presented here, however, do not belong to this class of defects but originate from contaminations from the residual gas. The chemical identity of these adsorbates could not be clarified. However, our model is of general applicability and, therefore, the knowledge about their chemical nature is not needed in this context. The adsorbates shown in Fig. 6(a) are imaged as protrusions due to a larger negative frequency shift above the species compared to the surrounding sub- 
strate. Upon decreasing the tip-sample distance by changing the detuning set point to $-13.8 \mathrm{~Hz}$, the adsorbates are imaged as depressions, some of which exhibit a bright rim as shown in Fig. 6(b). The bright rim is attributed to a large negative detuning, while the depression in the center corresponds to a smaller negative or even positive detuning. At a detuning set point of $-10 \mathrm{~Hz}$, the deviation from this set point is smaller compared to the situation at a detuning set point of $-13.8 \mathrm{~Hz}$, as shown in the line plots in Fig. 6(c). The corresponding $z$ positions are shown in Fig. 6(d).

Interestingly, with a detuning set point of $-13.8 \mathrm{~Hz}$, the tip first senses a smaller negative and even positive detuning, as shown in Fig. 6(c). This causes the feedback to further approach the tip toward the sample [see Fig. 6(d)]. When the tip has passed over the adsorbate, a sudden increase in the negative detuning is measured, which results in the retraction of the tip. ${ }^{23}$

\section{SUMMARY AND CONCLUSION}

In conclusion, we provide means for differentiating whether or not repulsive interactions are sensed in NC-AFM imaging. For each tip-sample interaction potential, one particular amplitude exists for which the change from attractive to repulsive interaction coincides with the change in sign in the $\Delta f$ curve. For large enough amplitudes (typically $>1 \AA$ depending on the tip-sample interaction decay length), the measurement of positive frequency shifts is a clear indication that the lower turning point of the oscillating tip enters into the repulsive regime. Based on this analysis, we discuss two different origins for contrast inversion. Contrast inversion between two different sample sites $\mathbf{A}$ and $\mathbf{B}$ is always associated with a crossing point in the corresponding $\Delta f(z)$ curves. This crossing point can have distinctly different origins. On the one hand, chemically different sample sites have different $\Delta f(z)$ curves that may have a crossing point, which can be in the attractive region of both sample sites. In the case of a crossing point in the attractive region, both constant height at different tip-sample distances and constantdetuning imaging at different detuning set points yield an inversion in imaging contrast. On the other hand, chemically identical sample sites can also lead to contrast inversion if the relative height of both sites is shifted, leading to two identical $\Delta f(z)$ curves that are shifted in $z$. In this case, the crossing point is necessarily in the region of negative slope of the $\Delta f(z)$ curve of the protruding sample site. Again, measuring in constant-height mode at two different sample sites clearly reveals contrast inversion. In constant-detuning mode, however, the region of contrast inversion is principally not accessible due to feedback loop constraints as the sign in the slope of the $\Delta f(z)$ curve changes. Constantdetuning imaging with slow feedback loop settings can, nevertheless, be performed and allows for penetrating into the repulsive regime while scanning over a protruding adsorbate. Our analysis reveals that the feedback loop severely alters the original information, which makes the interpretation of these constant-detuning experiments rather delicate.

\section{ACKNOWLEDGMENTS}

Financial support from the Deutsche Forschungsgemeinschaft (DFG) through an Emmy Noether grant is gratefully acknowledged. We thank Michael Reichling for fruitful discussions and critical comments. *kuehnle@uos.de

${ }^{1}$ G. Binnig, C. F. Quate, and C. Gerber, Phys. Rev. Lett. 56, 930 (1986).

${ }^{2}$ F. J. Giessibl, Science 267, 68 (1995).

${ }^{3}$ C. Barth, A. S. Foster, M. Reichling, and A. L. Shluger, J. Phys.: Condens. Matter 13, 2061 (2001).

${ }^{4}$ J. V. Lauritsen, A. S. Foster, G. H. Olesen, M. C. Christensen, A. Kühnle, S. Helveg, J. R. Rostrup-Nielsen, B. S. Clausen, M. Reichling, and F. Besenbacher, Nanotechnology 17, 3436 (2006).

${ }^{5}$ A. I. Livshits, A. L. Shluger, A. L. Rohl, and A. S. Foster, Phys. Rev. B 59, 2436 (1999).

${ }^{6}$ A. S. Foster, W. A. Hofer, and A. L. Shluger, Curr. Opin. Solid State Mater. Sci. 5, 427 (2001).

${ }^{7}$ S. Hirth, F. Ostendorf, and M. Reichling, Nanotechnology 17, S148 (2006).

${ }^{8}$ Y. Sugimoto, P. Jelinek, P. Pou, M. Abe, S. Morita, R. Pérez, and O. Custance, Phys. Rev. Lett. 98, 106104 (2007).

${ }^{9}$ K. Kobayashi, H. Yamada, T. Horiuchi, and K. Matsushige, Appl. Surf. Sci. 140, 281 (1999).

${ }^{10}$ T. R. Ramachandran, C. Baur, A. Bugacov, A. Madhukar, B. E. Koel, A. Requicha, and C. Gazen, Nanotechnology 9, 237 (1998).

${ }^{11}$ J. M. Mativetsky, S. A. Burke, R. Hoffmann, Y. Sun, and P. Grütter, Nanotechnology 15, S40 (2004).

${ }^{12}$ T. Arai and M. Tomitori, Appl. Surf. Sci. 157, 207 (2000).
${ }^{13}$ H. Özgür Özer, S. J. O’Brien, and J. B. Pethica, Appl. Phys. Lett. 90, 133110 (2007).

${ }^{14}$ M. Guggisberg, O. Pfeiffer, S. Schär, V. Barwich, M. Bammerlin, C. Loppacher, R. Bennewitz, A. Baratoff, and E. Meyer, Appl. Phys. A 72, S19 (2001).

${ }^{15}$ M. Reichling and C. Barth, Phys. Rev. Lett. 83, 768 (1999).

${ }^{16}$ R. Pérez, I. Štich, M. C. Payne, and K. Terakura, Phys. Rev. B 58, 10835 (1998).

${ }^{17}$ F. J. Giessibl, Phys. Rev. B 56, 16010 (1997).

${ }^{18}$ F. J. Giessibl, Rev. Mod. Phys. 75, 949 (2003).

${ }^{19}$ F. J. Giessibl and H. Bielefeldt, Phys. Rev. B 61, 9968 (2000).

${ }^{20}$ M. Abramowitz and I. A. Stegun, Handbook of Mathematical Functions with Formulas, Graphs, and Mathematical Tables, Applied Mathematics Series No. 55 (National Bureau of Standards, Washington, D.C., 1964).

${ }^{21}$ Obtained by numerical evaluation of Eq. (5).

${ }^{22}$ S. Wendt et al., Surf. Sci. 598, 226 (2005).

${ }^{23}$ See EPAPS Document No. E-PRBMDO-77-080819 for an illustration of the feedback loop effect. In these three Shockwave Flash movies, the tip movement over an adsorbate is shown with three different loop gain settings. These animations have been created using a simple detuning-distance curve based on a Morse potential as used for the simulations. Likewise, a Gaussian distribution is applied to the equilibrium distance for simulating a protruding adsorbate. For more information on EPAPS, see http://www.aip.org/pubservs/epaps.html. 